\title{
다양한 형태의 운동에 따른 족저압 변화의 체계적 고찰 *
}

\author{
최혁재 ${ }^{12)} \cdot$ 강현주 ${ }^{2)^{* *}}$
}

1) 근로복지공단 재활공학연구소 2) 순천향대학교

\begin{abstract}
Hyuk-Jae Choi • Hyun-Joo Kang. Systematic Review of Plantar Pressure Change in Varied Exercises. KINESIOLOGY, 2015, 17(4): 31-37. [PURPOSE] This study suggests effective guideline manuals prescribed for different exercise programs and appropriate sports medicine applications for preventing injury and improving physical activity through a systematic review of precedent literatures about plantar pressure. [METHODS] We selected Zijlstra et al's method (2007) of pre-study, which is based on precedent literature published by many researchers that have engaged in both domestic and international study. In this systematic review, we selected Zijlstra et al's model and then revised the appropriate key-words from precedent literature to our present study. [RESULTS] We selected nine studies located in research databases from 378 related articles. These studies were summarized in terms of inclusion and exclusion about analyzing plantar pressure in sports medicine. Results showed a significant number of relevant results in sport medicine and exercise fields through analyzing plantar pressure. Plantar pressure had significant differences between pre-test and post-tests such as balance, stability, and body posture. [CONCLUSIONS] The value of these plantar pressure-related studies demonstrates injury prevention when prescribed for normal people to be active in a safe manner. Additionally, these studies consider guidelines for foot movement as well as removing risk factors for injury and coaching exercise.
\end{abstract}

Key words : plantar pressure, exercise, normal person, injury 주요어 : 족저압, 운동, 정상인, 손상

\section{서론}

산업화와 더불어 소득수준이 증가함에 따라 사회 전 반적으로 삶의 질이 높아졌고 향상된 삶의 질을 공유하 기 위해 업무시간 이외의 기타 여가 및 휴식시간 동안 건강 유지와 증진을 위해 다양한 신체활동을 즐기는 인 구가 증가한다고 보고하고 있다(Ministry of Culture, Sports \& Tourism, 2005).

신체활동의 효과증진과 손상예방을 위해 생활체육, 임상, 스포츠의학, 재활의학, 물리치료, 생체역학, 인간공 학 등의 다양한 분야에서 관심을 가지고 있다. 신체활동 가운데 가장 간편하고 대중화된 걷기 운동을 수행하면 서 신체의 균형이나 안정성을 유지하는 기능은 신체활 동에 있어 매우 중요한 역할을 한다(Giacomozzi, 2010; Monteiro et al., 2010). 이러한 중요한 기능을 지속적으 로 유지하기 위해서는 족부를 포함한 하지의 여러 특징 을 고려하고 지속적인 관리가 필요하다. 최근 들어 인체
의 하지에 대한 연구 가운데 족부의 문제와 기능을 효 과적으로 진단하기 위해 임상이나 학술 분야에서 여러 연구들을 진행해오고 있다(Deepashini et al., 2014).

Teh et al.(2006)의 연구에 의하면 족저압 분석을 통해 하지의 근골격계 질환에 대한 수많은 원인을 다 양한 관점에서 접근할 수 있다고 보고하고 있다. 하지 만 족저압의 변화는 여러 가지 요인에 영향을 많이 받고 있다. 족저압 변화 요인에 대한 연구 가운데 Periyasamy et al.(2011)은 성별, 체중, 족부의 관절운 동범위에 따라 족저압의 변화에 대한 요인을 연구하 였고 Deepashini et al.(2014)는 기존에 발표된 여러 논문을 바탕으로 성별, 체중, 나이에 따라 족저압에 영향을 줄 수 있는 고찰연구를 진행하였다. 기존의 성 별, 나이, 체중, 관절운동범위 등의 변수에 대한 족저 압 연구는 많이 분석되어 왔으나 다양한 형태의 운동 을 통해 족저압의 변화를 체계적으로 살펴본 연구는 부족한 실정이다. 본 연구에서는 체계적 고찰연구를

* 이 연구는 2015년 순천향대학교 학술지원비와 미래창조과학부 한국연구재단 기초연구사업의 지원을 받아 수행됨(NRF-2013-R1A1A1013430).

** 교신저자: violethjk@hanmail.net 
통해 운동학 분야에 적용되는 족저압과 관련된 기존 연구를 바탕으로 분석해 가고자 한다.

따라서 본 연구의 목적은 다양한 형태의 운동과 족 저압의 변화와 관련된 선행연구를 체계적으로 고찰하 여 스포츠 현장이나 재활 분야에서 신체활동에 대한 효과성을 검증하고 운동처방에 필요한 중요한 자료를 제공하고자 한다.

\section{연구방법}

국내 및 국외에서 수많은 저자들에 의해 출판된 체 계적 논문 고찰연구(운동학 분야에서의 족저압)에 대한 문헌을 검색한 결과는 다음과 같다. 본 연구의 체계적 고찰연구에 관한 연구방법은 기존의 연구된 Zijlstra's model을 바탕으로 본 연구에 해당되는 중요단어로 수 정하여 진행하였다(Zijlstra et al., 2007). Zijlstra's model은 연구방법의 타당성을 입증하기 위해 3단계 의 연구 방법으로 진행하였고 족저압과 관련된 운동 효과의 체계적 고찰연구를 위해 밀접하게 연관이 있 는 단어를 내포한 포함범위(inclusion)와 제외단어를 포함한 제외범위(exclusion)를 구분하여 연구를 진행 하였다(Lee \& Kim, 2013).

첫 번째 단계는 족저압, 운동효과, 일반인, 무작위 추출, 타당도와 신뢰도가 높은 장비사용의 키워드를 중심으로 국외 논문 검색 사이트(PUBMED, SCOPUS, MEDLINE, SCIENDIRECT)와 국내 논문 검색 사이트 (국회도서관, KISS, DEPIA)에서 2001년부터 2015년 10 월까지 연구된 논문을 검색하였다.

두 번째 단계로 실험외적변인, 혼입 등을 배제하고 두 집단을 최대한 비슷하게 구성하기 위해 정해진 규칙
없이 대상자를 각각의 집단에 무작위로 할당하고 통제 하는 무선할당통제연구(RCTs: Randomized Controlled Trials)방법을 수행한 논문을 포함하였다.

마지막으로 세 번째 단계는 본 연구의 포함범위(indusion) 중 일반인을 대상으로 족저압 키워드를 중심으로 검 색하였다. 족압이 스포츠선수나 환자에 필요한 요소이 긴 하지만 본 연구에서는 전문적인 스포츠 활동이나 병적상태의 손상들을 제외하고 일반적인 운동에 대한 효과가 족압에 미치는 영향만을 살펴보고자 하였다.

세 가지 단계를 거쳐 운동학 분야의 족저압 분석연 구에 따른 운동효과에 대한 체계적 고찰연구 방법을 진행하였다.

\section{결과}

족저압과 운동과 관련된 체계적 고찰은 국내 및 국외 를 포함해 최종적으로 9 개의 논문을 고찰 하였다. 9 개의 논문은 포함범위와 제외범위에 따라 다양한 운동학 분 야에서 족저압을 분석한 논문을 <Table 1>에 제시하였 다. 최종적으로 선정한 9 개의 논문 중 국내논문은 국회 도서관의 검색사이트를 이용하여 첫 번째 선별작업(족저 압)을 통해 169 편의 논문을 검색하고 그 중 운동과 관련 된 논문 42 편으로 선별하고 42 편의 논문 중 제목과 요 약본에 대한 고찰을 통해 최종적으로 4 편을 선정하였다.

국외논문는 PUBMED, SCOPUS, MEDLINE, SCIEN $\mathrm{DIRECT}$ 의 문헌 검색를 통해 족저압(plantar pressure) 과 운동효과(exercise effect) 분류에서 209편 검색하고 다음으로 제목과 요약본을 통해 27편의 논문을 검색 하였다. 27편의 논문 중 심도 있는 고찰을 통해 일반 인, 무작위 추출 등 포함범위와 환자, 전문적인 운동선

Table 1. Summary of Literature Review

\begin{tabular}{|c|c|c|c|c|c|c|}
\hline $\begin{array}{l}\text { Au., } \\
\text { Date }\end{array}$ & Subjects & $\begin{array}{l}\text { Purpose of } \\
\text { the Study }\end{array}$ & Exercise Intervention & Exercise-type & Outcomes & Measurement \\
\hline $\begin{array}{l}\text { Mao, } \\
\text { D. W. } \\
(2006) \\
1\end{array}$ & $\begin{array}{l}16 \mathrm{P} . \\
\text { (M.8,F.8) } \\
(23.1 \pm 5.5 \\
\text { A.) }\end{array}$ & $\begin{array}{l}\text { Analyzing } \\
\text { PLPR } \\
\text { concerning the } \\
\text { five basic } \\
\text { movements of } \\
\text { Tai Chi }\end{array}$ & $\begin{array}{l}\text { All subjects } \\
\text { participated in three } \\
\text { trials of five Tai-Chi } \\
\text { movements, next three } \\
\text { trials of } 15 \mathrm{~m} \text { gait. } \\
\text { This study was not } \\
\text { designed as an } \\
\text { exercise intervention. }\end{array}$ & $\begin{array}{l}\text { Five basic } \\
\text { movements } \\
\text { of Tai Chi }\end{array}$ & $\begin{array}{l}\text { For analyzing PLPR, we } \\
\text { divided nine areas of the } \\
\text { plantar region. Distribution of } \\
\text { PLPR, and strengthening } \\
\text { around these parts, and } \\
\text { improving balance is effective } \\
\text { in the first toe and first } \\
\text { metatalsal. }\end{array}$ & $\begin{array}{l}\text { NovelPedar- } \\
\text { X, Munich, } \\
\text { germany } \\
\text { (insoletype) }\end{array}$ \\
\hline
\end{tabular}




\begin{tabular}{|c|c|c|}
\hline $\begin{array}{l}\text { Burn } \\
\text { field, } \\
\text { J. M. } \\
\text { (2007) }\end{array}$ & $\begin{array}{l}20 \mathrm{P} . \\
(\mathrm{M} 10, \mathrm{~F} 10) \\
(19<\mathrm{A},>60)\end{array}$ & $\begin{array}{l}\text { Analyzing } \\
\text { PLPR about } \\
\text { five exercises } \\
\text { of the } \\
\text { cadiovascular } \\
\text { system }\end{array}$ \\
\hline
\end{tabular}

2
Study divided two

GPs per ten persons. All subjects undertake five exercises for a minimum of five minutes and set borg scales from six to twenty score. This study was not designed an exercise intervention.
Walking and Peak pressures higher during running on walking $(253 \mathrm{kPa})$, running treadmill, (251kPa), elliptical (213kPa), stair climbing, elliptical training, recumbent biking forefoot. Biking pressures lower than all others $(\mathrm{P}<0.001)$. Pressures higher during running compared with all
NovelPedar-

$X$

Munich, $\begin{array}{ll}\text { climbing }(130 \mathrm{kPa}) \text { and } & \text { germany } \\ \text { recumbent }(41 \mathrm{kPa} ; \mathrm{P} \leq 0.001) & \text { in }\end{array}$ others $(\mathrm{P} \leq 0.001)$ in arch. Pressures were lowest during recumbent $(33 \mathrm{kPa} ; \mathrm{P}<0.001)$. Pressures were highest during walking $(215 \mathrm{kPa})$ and running (188kPa) in heel.

$\begin{array}{llllll}\text { Hong } & \text { 20P. } & \text { Analyzing } & \text { Study divided two } & \text { Modern and Ratio of peak pressures } & \text { TPScan(Biom } \\ (2009) & \text { (F.20) } & \text { PLPR, } & \text { GPs as modern and traditional } & \text { between fore and hind-foot: } & \text { echanics Co., } \\ \text { (39.1 } \pm 7 \text { A.) } & \begin{array}{l}\text { physical } \\ \text { strength, and }\end{array} & \begin{array}{l}\text { traditional GP per ten pilates with } \\ \text { persons. All subjects mat }\end{array} & \begin{array}{l}\text { results of traditional GP is } \\ \text { more effective than modern. }\end{array} & \text { Korea) } \\ \text { (plate type) }\end{array}$
pain in pilates spend time of exercise mat exercises, from fifty to sixty both modern minutes, and three and traditional times per week for method twelve weeks.

Evaluated two times both pre and post-test after twelve weeks.

\begin{tabular}{|c|c|c|c|c|c|c|}
\hline $\begin{array}{l}\text { Jeon } \\
\text { (2010) }\end{array}$ & $\begin{array}{l}\text { 45P. } \\
(25 \pm 4.5 \mathrm{~A} .)\end{array}$ & $\begin{array}{l}\text { Confirming } \\
\text { effect of } \\
\text { alteration of } \\
\text { body type, } \\
\text { and PLPR, } \\
\text { and trunk } \\
\text { muscle activity } \\
\text { in bridge } \\
\text { exercise }\end{array}$ & $\begin{array}{l}\text { Study progressed } \\
\text { three sets of } 15 \\
\text { repetitions at three } \\
\text { times per week for } \\
\text { eight weeks. } \\
\text { Evaluated five times } \\
\text { in pre, 2th, 4th, 6th, } \\
\text { and lastly 8th. }\end{array}$ & $\begin{array}{l}\text { Bridge } \\
\text { exercise: } \\
\text { abdominal } \\
\text { hollowing } \\
\text { method }\end{array}$ & $\begin{array}{l}\text { PLPR of both 1st\&5th } \\
\text { metatarsal and heel regions: } \\
\text { there is a bit of a significant } \\
\text { difference. } \\
\text { Bridge exercise is effective for } \\
\text { stability of trunk, and optimal } \\
\text { distribution of PLPR, and } \\
\text { improvement of physical } \\
\text { balance, and symmetric weight } \\
\text { distribution. }\end{array}$ & $\begin{array}{l}\text { GPS400, Italy } \\
\text { (plate type) }\end{array}$ \\
\hline $\begin{array}{l}\text { Lee } \\
\text { (2011) }\end{array}$ & $\begin{array}{l}\text { 32P. } \\
\text { (M.18,F.18) } \\
(20<A .>35)\end{array}$ & $\begin{array}{l}\text { After } \\
\text { application of } \\
\text { selective eve } \\
\text { movement } \\
\text { program, } \\
\text { confirming } \\
\text { effects of } \\
\text { static and } \\
\text { dynamic } \\
\text { balance, and } \\
\text { PLPR }\end{array}$ & $\begin{array}{l}\text { Study divided three } \\
\text { GPs per twelve } \\
\text { persons at same } \\
\text { gender ratio. We } \\
\text { evaluated three times } \\
\text { at pre, } \\
\text { after-five-exercises, } \\
\text { and lastly } \\
\text { after-nine- exercises. } \\
\text { The first pre-test } \\
\text { intervention is five } \\
\text { treatments then } \\
\text { second test. The } \\
\text { second intervention is } \\
\text { three times per week } \\
\text { for three weeks, total } \\
\text { nine treatments to } \\
\text { next post-test. }\end{array}$ & $\begin{array}{l}\text { 1st GP: } \\
\text { saccadic, 2nd } \\
\text { GP: } \\
\text { vestibulo-ocu } \\
\text { lar, } \\
\text { 3th GP: } \\
\text { pursuit } \\
\text { vergence } \\
\text { exercise }\end{array}$ & $\begin{array}{l}\text { Asymmetric index of PLPR: } \\
\text { There are significant } \\
\text { differences in all GPs. Eve } \\
\text { movement exercises are } \\
\text { effective for distribution. }\end{array}$ & $\begin{array}{l}\text { Gaitview } \\
\text { AFA-50, } \\
\text { Alfoots, } \\
\text { Korea(plate } \\
\text { type) }\end{array}$ \\
\hline $\begin{array}{l}\text { Willems, } \\
\text { T. M. } \\
6 \text { (2012) }\end{array}$ & $\begin{array}{l}52 \mathrm{P} . \\
(\mathrm{M} .36, \mathrm{~F} .16) \\
(23<\mathrm{A} .>62)\end{array}$ & $\begin{array}{l}\text { When long } \\
\text { distance }(20 \mathrm{~km}) \\
\text { running } \\
\text { exercise, study } \\
\text { of factors of }\end{array}$ & $\begin{array}{l}\text { Study did not divide } \\
\text { GP and evaluated two } \\
\text { times- first pre-test, } \\
\text { next post-test after } \\
\text { 20km running at the }\end{array}$ & $\begin{array}{l}\text { long } \\
\text { distance(20k } \\
\text { m) running } \\
\text { exercise }\end{array}$ & $\begin{array}{l}\text { Patterns of PLPR changed } \\
\text { variously after } 20 \mathrm{~km} \text { running: } \\
\text { 1. Loading increased: } \\
\text { medial-heel, mid-foot, and } \\
\text { metatarsal part. }\end{array}$ & $\begin{array}{l}\text { Footscan } \AA_{\text {,Rs }} \\
\text { ScanInternati } \\
\text { onal } \\
\text { (platetype) }\end{array}$ \\
\hline
\end{tabular}


Willems, 52P. When long Study did not divide long $\quad$ Patterns of PLPR changed Footscan®,Rs

T. M. (M.36,F.16) distance $(20 \mathrm{~km})$ GP and evaluated two distance $(20 \mathrm{k}$ variously after $20 \mathrm{~km}$ running: ScanInternati

$6(2012)$

$6(2012) \quad(23<\mathrm{A} .>62)$

$\begin{array}{ll} & \\ \text { Wong, } & \text { 10P. } \\ \text { S. H. } & \text { (M.10) }\end{array}$

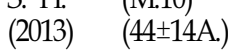

7

\begin{tabular}{|c|c|c|}
\hline $\begin{array}{l}\text { Kwon } \\
\text { (2014) }\end{array}$ & $\begin{array}{l}16 \mathrm{P} . \\
(\mathrm{F} .16) \\
(31.9 \pm 6.5 \mathrm{~A} .)\end{array}$ & $\begin{array}{l}\text { Confirming } \\
\text { effect of PLPI } \\
\text { and alignmen } \\
\text { of sagittally } \\
\text { vertical plane } \\
\text { and forward } \\
\text { head posture } \\
\text { in pilates } \\
\text { reformer } \\
\text { exercise }\end{array}$ \\
\hline $\begin{array}{l}\text { Richard } \\
\text { Marks } \\
(2014)\end{array}$ & $\begin{array}{l}\text { 9P. } \\
(\mathrm{M} .5, \mathrm{~F} .4) \\
(22<\mathrm{A} .>28)\end{array}$ & $\begin{array}{l}\text { Comparative } \\
\text { study of } \\
\text { PLPR using } \\
\text { various } \\
\text { devices for } \\
\text { rehabilitation }\end{array}$ \\
\hline
\end{tabular}

9 biomechanica features through analyzing PLPR, and Chi push-hand exercise, study next post-test after m) running exercise Confirming Ten subjects fulfilled ground reaction designer force in Tai intervention. two push-hand movements both and without opponent at regular pace. This study was not

Tai Chi
push-hand exercise (opponent present or not) intervention.

Study divided two GPs pilates
Study divided two GPs pilates GP per eight persons. All subjects spend an exercise time of fifty minutes, three times per week, for eight weeks. We evaluated PLPR two times- both pre and post-test after approximately eight weeks.

Study did not divide
GP and set resistance at 6 of a maximal 20 score and set self selective pace at all methods of exercises. In treadmill, we set resistance at 9 , and slope at the 5 value. In ground walking, we set a self selective speed and distance of six meters. This study was not designed as an exercise intervention.

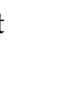

1. Loading increased: medial-heel, mid-foot, and metatarsal part.

The largest value is in the 1st NovelPedartoe region of the nine total $X$ regions in the plantar region Munich at push-hand exercise that is (insoletype) useful for strengthening the 1st toe and improving balance, but has a significant caution due to diabetes peripheral neuropathy onal (platetype)
Left·right \& fore-hindfoot ratio Shisei

of PLPR difference:

1. Ratio of left-right decreased: Footpressure, every GPs

2. Ratio of fore-hindfoot decreased (10\%): only exercise GP

Plates reformer is useful for optimal body posture and improvement of foot balance japan (plate type)

\begin{tabular}{|c|c|}
\hline $\begin{array}{l}\text { Stationary } \\
\text { bike, stair } \\
\text { stepper, } \\
\text { elliptical } \\
\text { trainer, } \\
\text { treadmill, } \\
\text { ground } \\
\text { walking. }\end{array}$ & $\begin{array}{ll}\text { Peak pressure, pressure time } & \text { F-Scan } \\
\text { integrals, and contact area in } 4 & \begin{array}{l}\text { insoles } \\
\text { regions of the foot: }\end{array} \\
\text { (Tekscan, } \\
\text { 1. stationary bike: lowest PLPR South } \\
\text { in all regions } & \text { Boston, MA) } \\
\text { 2. stair stepper: lowest mean } & \text { (plate type) } \\
\text { peak pressure of all } & \\
\text { weight-bearing exercises } & \end{array}$ \\
\hline
\end{tabular}

AU.=Author, P.=Persons, M=Male, F=Female, A=Age, PLPR=Plantar Pressure, GP=Group

수 등의 제외범위를 고려하여 총 5편의 논문을 선정 하였다. 최종적으로 선정된 9편의 논문은 출판시기에 따라 가장 나중에 발간된 순서로 번호를 부여하였다.

전체 논문에서 다루어진 다양한 운동은 안구운동 1 편, 필라테스 운동 2편, 교각운동 1편, Tai Chi 운동 2 편, 5 가지의 기구를 이용한 유산소운동 1 편, 4 가지 기 구운동과 평지보행을 적용한 재활운동 1 편, 마지막으로 $20 \mathrm{~km}$ 의 장거리달리기 1 편으로 나타났다. 각각의 연구 에서 족저압 분석을 위해 사용된 장비는 인솔 타입(3 편)과 발판 타입(6편)으로 나타났다. 대상자는 모두 정 상 성인으로 선정하였고 동일하게 성비율을 정한 연구
(연구 1, 2, 5)는 3편으로 나타났다. 모든 연구에서 대 상자의 연령은 만 20세 이상 65 세 이하로 심각한 수술 이나 특정한 질병이 없는 정상인을 대상자로 선정하였 다. 연구 1에서 다루어진 Tai Chi 운동의 기본적인 5가 지 동작과 $15 \mathrm{~m}$ 의 보행을 적용하고 족저부 9 개 영역을 구분하여 족저압을 분석하였다. 분석결과는 엄지발가락 과 발허리뼈 부위에 부하가 많이 발생하고 보행동작과 기본적인 5 가지 동작에 대한 족저압은 많은 차이를 나 타냈다. 족저압의 부하정도와 비교를 통해 운동적용과 손상을 예방하는 중요한 연구이다(Burnfield et al., 2007; Mao et al., 2006; Lee et al., 2011). 
연구 1과 7에서 다루어진 Tai Chi 운동은 전족부위와 엄지발가락 부위의 과도한 부하에 금기시 되는 당뇨환자 나 말초신경환자에 주위를 요구하고 있다. 연구 3에서 다루어진 필라테스 운동은 Jane(2002)의 연구에서 다루어 진 현대적인 필라테스 매트 운동방식과 Joseph \& William(2002)의 전통적인 방식에 따른 족저압의 압력비 율을 종속변수로 선정하였다. 현대적인 방법과 전통적인 운동방법의 세부사항은 연구 3의 운동방법에 자세히 소 개되어져 있다(Jane et al., 2008; Joseph \& William, 2002). 족저부위의 전방과 후방의 중심선을 기준으로 구 분하고 전방과 후방부위의 족저압의 비율은 전통적인 방 식을 적용한 군에서 더 효과적인 결과를 나타냈다. 필라 테스 운동을 다룬 연구 8은 전통과 현대적인 매트운동이 아닌 필라테스 리포머 운동기구를 사용하였다(Mao et al., 2006; Wong et al., 2013; Hong et al., 2009).

연구 8 에서는 연구 3 에서 다루어진 족저압 비율을 종속변수로 선정하였지만 추가적으로 좌·우에 대한 비 율을 종속변수로 선정하였다. 연구 3에서 8주 동안의 필라테스 운동 적용 시 좌·우 비율은 통제군과 운동군 에서 모두 향상 되었지만 앞-뒤 비율은 유일하게 운동 군에서 감소되었다. 연구 4 에서는 교각운동 중 갈고리 형태로 바로 누운 자세에서 배꼽을 내측상방으로 당기 며 척추 만곡 각도 유지, 갈비빼의 움직임 고정, 마지 막으로 자연스러운 호흡을 하며 복직근의 수축을 확인 하는 할로잉(hollowing) 운동 자세에 중점을 두어 8 주 동안 적용하였다. 연구 4 의 결과는 양쪽 제 1 과 5 번째 발허리뼈와 발꿈치뼈의 족저압에서 유의한 차이를 나 타냈다(Kwon, 2014; Moon et al., 2013; Jeon, 2010).

결과에서는 교각운동이 체간안정, 신체균형, 족저압의 대칭적인 분포에 효과적이라고 보고하고 있다. $20 \mathrm{~km}$ 의 장거리 달리기 운동방법을 적용한 연구 6 은 $20 \mathrm{~km}$ 달리 기 후 부하가 증가되는 부위와 부하가 감소되는 부위를 결과에 나타내었고 이러한 결과를 바탕으로 장거리 달 리기 운동 시 손상 위험요소를 족저압 변화 패턴을 통 해 발견하였다(Willems et al., 2012). 연구 2와 연구 9는 기구를 이용한 운동을 연구방법으로 선정하였다. 연구 2 에서는 5 가지 운동 중 족저압에 대한 적절한 압력감소 는 자전거, 스텝퍼 운동이 효과적이고 후족부의 높은 압 력, 힘을 분산시키기 위해서는 걷기나 달리기 운동보다 는 자전거, 일립티컬, 스텝퍼 운동이 효과적임을 보고하
였다(Burnfield et al., 2007). 연구 9에서 사용된 4가지 운동장비와 평지보행에 대한 결과는 고정식 자전거 운 동 시 전체적인 족저 영역에서 족저압이 감소하고 스텝 퍼 운동은 체중지지가 가장 높았고 전족부와 중족부의 지지시간이 증가했다(Marks et al., 2014).

\section{고찰}

운동학 분야에서 족저압에 대한 변화 및 패턴에 대 한 분석은 운동의 효과와 손상 예방을 위해 중요한 역할을 한다. 족저압을 분석하는 장비와 분석 방법, 족저압과 동시에 신체 족부의 운동학 및 운동 형상학 적 분석이나 손상 기전을 연구하는 다양한 방법이 있 다. 본 연구를 통해 족저압이 운동학 분야에서 다양하 게 연구되는 방법과 적용 사례에 대한 고찰을 통해 운동처방 시 중요한 정보를 제공하고자 하였다.

고찰 연구로 선정된 9편의 논문은 족저압 측정 도 구로 인솔타입과 발판타입으로 구분할 수 있다. 인솔 타입은 인솔과 신발을 동시에 착용하고 다양한 지면 에 대한 연구가 가능하지만 발판타입의 측정 장비는 맨발(barefoot)상태로 대상자 족부의 고유한 특성에 대한 연구에 효과적이다. 인체 족부에 대한 고유의 특 성을 연구하기 위해서는 맨발상태(barefoot)로 측정하 는 것이 신발이나 인솔에 대한 변수를 통제할 수 있 다. 그리고 인솔타입의 연구는 신발의 특성과 인솔의 재질 등 여러 가지 변수에 의해 족부에 영향을 미치 기 때문에 변수에 대한 신중한 통제방법이나 중재가 필요할 것으로 사료 된다.

연구 1,2 , 7은 인솔 타입의 측정 장비를 이용하였 지만 발판타입의 연구를 동시에 진행하여 인솔타입과 발판타입의 차이점에 대한 연구가 필요하다고 사료된 다. 이와 마찬가지로 발판타입의 측정 장비를 사용한 연구 $3,4,5,6,8$,9도 인솔 타입의 연구가 필요할 것 으로 여겨진다. 인솔과 발판을 통한 족저압 측정에 대 한 장단점이 존재하지만 측정되는 변수는 동일하게 얻 을 수 있으므로 각각의 장비의 특성을 고려해서 앞으 로 진행되는 연구에 적절하게 적용할 필요성이 있다. 9편의 논문 중 족저압에 대한 종속변수를 유일하게 채택한 연구도 있지만 주로 족저압과 동시에 균형능 
력, 지면반발력, 자세측정, 체력, 통증, 체간근 활성도 등을 분석하여 다른 변인과의 상관성을 살펴보았다.

운동학 분야에 적용을 위해 족저압과 다양한 측정 변수를 통한 연구가 운동 효과에 대한 결론을 객관적 으로 뒷받침 할 수 있는 중요하고 필요한 연구라고 사료된다. 측정 부위를 구분할 때에는 족저압을 측정 하기 위해 2 개 영역인 전·후족부, 3 개 영역인 전·중. 후족부, 6 개 영역, 최대 9개 영역으로 세분하여 나눌 필요도 있을 것으로 판단된다.

세분화된 족저 부위 구분은 다양한 변수를 효과적으 로 분석하는 데 용이하고 측정 장비회사에 제공한 프 로그램이 각각의 영역을 분석하는데 효과적인 방법을 제공한다. 연구 3,5 는 비율, 비대칭 지수만을 분석한 경우 영역을 2 3개 정도 구분하였고 연구 $1,2,7$ 은 전 체 구분 영역을 6 9개로 구분하였다. 9개의 구분영역 은 족저압에 대한 다양한 변수를 측정 할 수 있다. 예 를 들어 족저압 $(\mathrm{kPa})$, 최대 힘 $(\mathrm{N})$, 접촉영역 $\left(\mathrm{cm}^{2}\right)$, 접촉 시간(\%cycle) 등이 측정 가능하고 많은 영역으로 구분 한 장비는 인솔타입의 Novel Pedar-X에서 제공한 프로 그램을 사용하는 공통점이 있었다. 다수의 측정 장비를 통한 족저압 분석은 여러 신체활동 후 족저 부위의 변 화를 가장 잘 나타내준다. 9편의 고찰연구들 중 긍정적 인 변화와 유의한 차이를 나타낸 결과로 운동의 효과 를 입증할 수 있었다. 운동 처방 시 족저압 변수에 대 한 변화 및 패턴을 고려하고 대상자에게 적용할 심도 있는 중재가 필요할 것으로 사료된다.

\section{결론}

족저압에 대한 체계적인 고찰연구를 통해 다양한 운동 시 족저압에 대한 긍정적인 변화와 패턴분석이 어느 정도 가능했고 운동처방 시 족저압에 대한 중요 성과 족저압을 고려한 다양한 운동에 대한 정보를 제 공한 중요한 자료라고 사료된다. 향후에는 일반인을 대상으로 한 걷기 등의 신체활동 뿐만 아니라 스포츠 선수들에게 있어서도 기량을 높이기 위한 수단과 부상 없이 안전하고 효율적으로 수행하기 위해서는 족저압 에 대한 정밀하고 다각적인 연구가 필요할 것이다.

\section{참고문헌}

Burnfield, J. M, Jorde, A. G., Augustin, T. R, Augustin, T. A, \& Bashford, G. R. (2007). Variations in plantar pressure variables across five cardiovascular exercises. Medicine and Science in Sports and Exercise, 39(11): 2012-2020.

Deepashini, H., Omar, B., Paungmali, A., Amaramalar, N. Ohnmar, H., \& Leonard, J. (2014). An insight into the plantar pressure distribution of the foot in clinical practice: Narrative review. Polish Annals of Medicine, 21(1): 51-56.

Giacomozzi, C. (2010). Appropriateness of plantar pressure measurement devices: a comparative technical assessment. Gait Posture, 32(1): 141-144.

Hong, S. M., Jung, S. D., \& Yang, J. H. (2009). Effects of health-related fitness, foot pressure, rating of perceived pain on Pilates matwork type in mid-aged women teachers. The Korean Journal of Physical Education, 48(4): 681-690.

Jane, H. (2002). UTS pre-plates matwork instructor's manual. NSW, Sydney: University of Technology, Sydney.

Jeon, H. Y. (2010). The Effects of a Bridging Exerciseon Body Shape Changes and Foot Pressure Distribution. [Master's degree thesis]. Graduate School, Daegu University Gyeongbuk, Korea. Joseph, H. P., \& William, J. M (2002). Your Health $\mathcal{E}$ Return to Life Through Contrology. Allan Menezes and The Pilates Institute of Australasia.

Kwon, J. E. (2014). The Effects of Pilates Reformer Exercise On Women's Foot Pressure, Sagittal Alignment and Forward Head Posture. [Master's degree thesis]. The Graduate School of Chung-Ang University, Korea.

Lee, G. C., Kim, C. H., Kim, M. J., Jung, G. W., Lee, J. C., Kim, J. H., Kim J. S., \& Bae W. S. (2011). Effects of the Selective Eye Movement Program on the Foot Pressure 
and Balance in Normal Adults. Journal of the korean society of medicine, 6(4): 425-436.

Lee, Y. H., \& Kim., K. T. (2013). Tai Chi as a Fall Prevention Intervention: An In-depth Literature Review of Randomized Controlled Trials and Suggestion to Taekwondo. KINESIOLOGY, 15(1): 1-11.

Mao, D. W., Li, J. X., \& Hong, Y. (2006). Plantar pressure Distribution During Tai Chi Exercise. Archives of Physical Medicine and Rehabilitation, 87(6): 814-820.

Marks, R., Beran, B., Long, J., Canseco, K, Grice, S., Szabo, A., Wang, Y., \& Harris, G. (2014). Plantar pressure analysis during rehabilitative exercise. Critical Reviews in Physical and Rehabilitation Medicine, 26(3-4): 193-201.

Ministry of Culture, Sports and Tourism(MCST) (2005). Sport white paper.

Monteiro, M., Aranha, J., Neves e Castro, M, Sousa M., \& Moreira, M. (2010). Influence of obesity and sarcopenic obesity on plantar pressure of postmenopausal women. Clin Biomech, 25(5): 461-467.

Moon, H. J., Jo, S. H., \& Goo, B. O. (2013). Difference of Trunk Muscles Activity during Hollowing vs Bracing Contraction in
Various Position. Journal of the korean society of physical medicine, 8(1): 11-18.

Periyasamy, R., Mishra, A., Anand, S., \& Ammini A. C. (2011). Preliminary investigation of foot pressure distribution variation in men and women adults while standing. Foot, 21(3): 142-148

Teh, E, Teng, L. F. Acharya, U. R., Ha, T. P. Goh, E., \& Min, L. C. (2006). Static and frequency domain analysis of plantar pressure distribution in obese and non-obese subjects. J Bodyw Mov Ther, 10(2): 127-133.

Willems, T. M., Ridder, R. D., \& Roosen, P. (2012). The effect of a long-distance run on plantar pressure distribution during running. Gait and Posture, 35(3): 405-409.

Wong, S. H., Ji, T., Hong, Y., Fok, S. L., \& Wang, L. (2013). Foot forces induced through Tai Chi push-hand exercises. Journal of Applied Biomechanics, 29(4): 395-404

Zijlstra, G. A., van Haastregt J. C., van Rossum, E., van Eijk, J. T., Yardley, L., \& Kempen, G. I. (2007). Interventions to reduce fear of falling in community-living older people: A systematic review. Journal of American Geriatric Society, 55: 603-615. 
